\title{
Postnatal development of Blake's pouch cyst: a case report and new insight for its pathogenesis
}

\author{
Seiichiro Hirono • Daisuke Ito • Hisayuki Murai • \\ Masayoshi Kobayashi • Maiko Suyama • \\ Katsunori Fujii • Naokatsu Saeki
}

Received: 9 April 2014 / Accepted: 27 May 2014 / Published online: 7 June 2014

(C) Springer-Verlag Berlin Heidelberg 2014

\begin{abstract}
Blake's pouch cyst (BPC), a rare cystic malformation in the posterior fossa, is believed to be caused by the congenital expansion of the posterior membranous area that normally regresses during embryogenesis. However, due to the wide spectrum of the onset pattern and age of patients, the natural history and the pathogenesis are poorly understood. The authors describe the case of a girl who admitted with headache and right abducens nerve paresis at the age of 3 years and 10 months. Magnetic resonance (MR) imaging demonstrated a tetraventricular hydrocephalus, an open aqueduct, and a posterior fossa cyst compatible with BPC. Multiple tumors were also noticed in the ventricular wall. Tumor biopsy and an endoscopic third ventriculostomy were performed. Intraoperative observation confirmed the BPC, and pathological diagnosis was pilomyxoid astrocytoma. In retrospect, MR imaging was performed twice in the past, at the age of 8 months and again at 22 months, and no anomaly was detected, suggesting that Blake's pouch was once regressed. Therefore, a BPC in this patient was certainly developed after her second or third year of life. The ventricular tumors may influence the cerebrospinal fluid (CSF) absorption, which triggered the reexpansion of BPC from the possible remnant of Blake's pouch. This is a rare but important report providing evidence that in addition to the classic congenital BPC in which the remnant of Blake's pouch remains persistent, there could be postnatal or secondary BPC, which develops after birth. Possible mechanisms include that the remnant of Blake's pouch, which originally disappears, may re-expand postnatally in
\end{abstract}

S. Hirono $(\varangle) \cdot$ D. Ito $\cdot$ H. Murai $\cdot$ M. Kobayashi $\cdot$ N. Saeki Department of Neurological Surgery, Chiba University Graduate School of Medicine, 1-8-1 Inohana, Chuoku, Chiba 260-8670, Japan e-mail: seiichirohirono@chiba-u.jp

M. Suyama $\cdot$ K. Fujii

Department of Pediatrics, Chiba University Graduate School of Medicine, 1-8-1 Inohana, Chuoku, Chiba 260-8670, Japan association with unknown trigger or a change in CSF dynamics or absorption.

Keywords Blake's pouch cyst $\cdot$ Hydrocephalus $\cdot$ Endoscopic third ventriculostomy $\cdot$ Pathogenesis

\section{Introduction}

Cystic malformations in the posterior fossa in children have been observed, often using several neuroimaging modalities. These cystic anomalies are generally classified into DandyWalker complex (DWC) and arachnoid cysts, which are simply collections of cerebrospinal fluid (CSF) covered by arachnoidal cells without communication to the subarachnoid space. DWCs include the Dandy-Walker malformation (DWM), Dandy-Walker variant (DWV), and mega-cisterna magna (MCM).

Blake's pouch cyst (BPC), described firstly in 1900 by Blake [1], is a rare posterior fossa cyst characterized by posterior ballooning of the posterior membranous area into the cisterna magna [2]. It has been reported as a distinct entity within the DWC [3] and recently is thought to be secondary to a congenital persistence of Blake's pouch caused by failure of spontaneous perforation of the foramen of Magendie during embryogenesis [2]. Since its first description in 1996, there have been reports of many patients with BPC, presenting a wide variety of symptoms ranging from asymptomatic to noncommunicating hydrocephalus in children, to gradually progressive communicating hydrocephalus in the elderly.

Neurosurgical interests in this rare malformation are raised, especially regarding its mechanisms and pathophysiology, because the age of patients with BPC ranges broadly from the newborn to the elderly [4]. Although the congenital formation of BPC has been proposed [2] and increasing awareness of this anomaly has facilitated the detection of this cyst 
even during the prenatal period using ultrasonography [5], the reason for some adults and even elderly patients reported with $\mathrm{BPC}$ remains enigmatic. Apparently, little is known about the natural history of BPC and its associated hydrocephalus.

Here, we report a case of BPC in a 3-year-old girl who presented with communicating hydrocephalus combined with BPC and ventricular tumors. Interestingly, she presents obvious evidence that BPC occurred more than 22 months after birth. This has profound implications for its pathophysiology and the possible therapeutic choices.

\section{Case report}

History, pre-onset radiography, and examination

The patient was originally followed prenatally because of abnormal nuchal translucency. Ultrasonography revealed no abnormality in the posterior fossa. Because delayed development and chromosomal abnormality were detected after birth, a pediatrician followed her routinely. Magnetic resonance (MR) imaging was performed at the age of 8 months and again at 22 months and no anomaly or cyst formation was observed in the posterior fossa (Fig. 1). However, at the age of 3 years and 10 months, she was admitted to our institution with headache and nausea, and neurological examination revealed a right abducens nerve paresis. MR images at admission revealed a moderate tetraventricular hydrocephalus, an open aqueduct, and a posterior fossa cyst located just beneath the vermis (Fig. 2a). The choroid plexus of the fourth ventricle protruded into the cyst cavity (Fig. 2b). No other anomaly including cerebellar rotation or vermian hypoplasia was demonstrated. These findings were completely compatible with BPC. Additionally, multiple tumors were noticed in the ventricle wall (Fig. 2c).

\section{Surgical management}

We performed a tumor biopsy and inspected the region around the BPC endoscopically, followed by an endoscopic third ventriculostomy (ETV) to manage the hydrocephalus. As MR imaging demonstrated, the outlet of the fourth ventricle was covered and obstructed by the cyst wall with a protrusion of the choroid plexus into the cyst cavity (Fig. 3a). There was a small pore on the midline of the cyst wall, which was compatible with the increased pressure of the ventricles because of the hydrocephalus and the consequent fenestration. The foramina of Luschka were open. Although the Liliequest's membrane at the basal cistern was tough, ETV was successfully performed and the hydrocephalus was well managed in the acute postoperative days. However, bacterial meningitis was encountered and secondary hydrocephalus was induced, which necessitated a ventriculoperitoneal shunt 1 month after ETV. During the shunt surgery, an endoscopic observation confirmed the patency of the

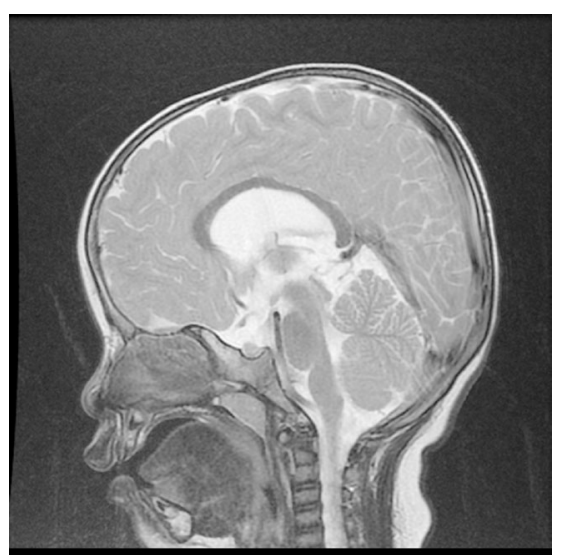

Fig. 1 Sagittal T2-weighted MR image at age of 22 months verifying no anomaly or cyst formation in the posterior fossa

opening at the floor of the third ventricle. Pathology of the tumor confirmed the diagnosis of pilomyxoid astrocytoma (Fig. 3b), and adjuvant chemotherapy based on vincristine and carboplatin was continued.

\section{Discussion}

BPC, a cystic malformation in the posterior fossa, is thought to be caused by the absence of spontaneous regression of Blake's pouch. In normal subjects, the Blake's pouch disappears with leaving a median opening, Blake's metapore, considered as the precursor of the foramen of Magendie [2]. Although approximately 20 cases have subsequently been reported in the literature, the incidence of this rare malformation is not well known, and neither its pathogenesis nor natural history is fully understood.

Diagnosis of this anomaly is mainly based on its radiological features. The main characteristic is the cyst communicating with the fourth ventricle with protrusion of the choroid plexus into the cyst. Although the vermis often has slight hypoplasia because of the mass effect, neither cerebellar rotation nor hypoplasia is observed. The confluence of sinuses is located in the normal position. These features not only reflect the embryological background of this malformation but are also useful for differential diagnosis from DWC, DWM, and MCM. Our case completely matched these features and was confirmed by intraoperative neuroendoscopic observation through the aqueduct and the fourth ventricle.

According to past case reports, hydrocephalus is the most common condition in BPC. However, there are some asymptomatic cases with BPC, which were discovered incidentally after routine examination by ultrasonography [6] or MR imaging [3]. Furthermore, antecedent triggers such as meningitis existed in some [4], showing that the clinical presentation of BPC differs among patients. 

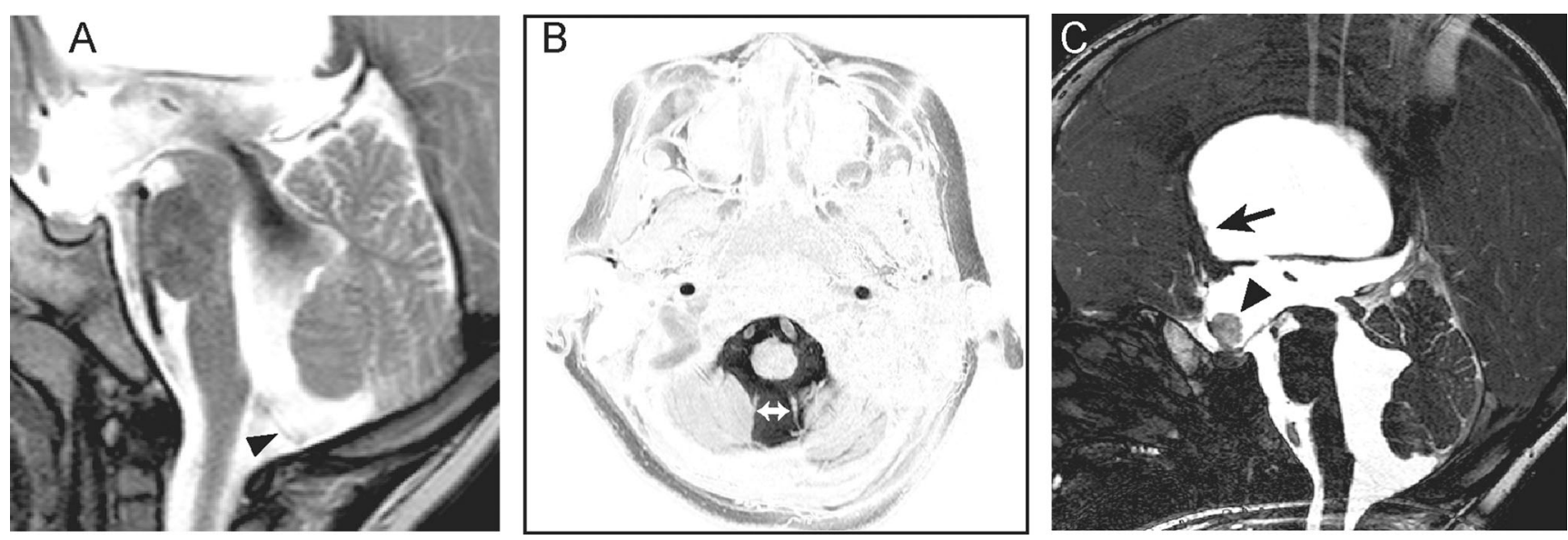

Fig. 2 Sagittal T2-weighted MR image at age of 3 years and 10 months demonstrating a cyst wall (arrowhead) below the vermis expanding to the subarachnoid space (a). Note the absence of any cerebellar or vermian anomaly and the normal position of the sinus confluence. The choroid

In addition to the wide variety of BPC symptoms, the age at diagnosis is diverse. The majority of patients with BPC are infants $[4,7]$. Recently, greater awareness of this entity has increased the rate of prenatal diagnosis of BPC by obsteric ultrasonography $[5,8]$, which is consistent with the proposed pathogenesis of BPC. By contrast, interestingly, there are some adult patients who are diagnosed with normal pressure hydrocephalus-like initial symptoms [3, 4]. One question raised, especially with those adult patients, was has BPC existed since the failure of Blake's pouch's regression during embryogenesis, with a long asymptomatic period?

The present case, which shows clear evidence that BPC developed a few years after birth, clearly suggests that some BPC might occur postnatally. Our case is unique in the sense that repeated MRI was performed before the diagnosis of BPC, verifying that BPC developed in the second or third year of her life, not during gestation. We speculate that in addition to the plexus of the fourth ventricle (arrow) protrudes into the cyst (b). Multiple tumors in the lateral ventricle wall (arrowhead) and a suprachiasmatic lesion (arrow) were observed (c)

classic congenital BPC in which the remnant of Blake's pouch remains persistent, there could be postnatal or secondary BPC, which develops after birth for some as yet unknown reason.

Another question raised by our case was how does postnatal or secondary BPC occur? Possible mechanisms include that the remnant of Blake's pouch, which originally disappears, may re-expand after birth with some unknown triggers or a change in CSF dynamics or absorption (Fig. 4, modified from [2]). Even if the remnant of Blake's pouch persists and the foramen of Magendie is not completely formed, CSF can circulate through the foramina of Luschka or the small Blake's pore to reach the subarachnoid space; therefore, the patient does not develop hydrocephalus, which is similar to the situation in the first 2 years of our patient. Subsequently, deterioration of the CSF absorption system was induced by the multiple tumors in our case and CSF flow via foramina of Luschka, or the small pore could no longer compensate, which

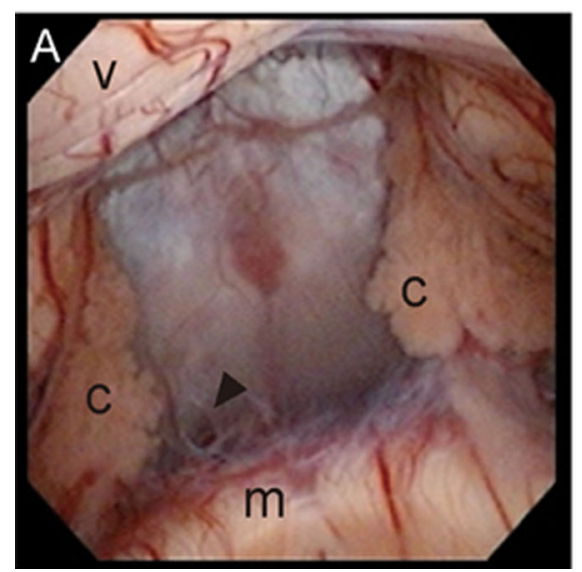

Fig. 3 Intraoperative findings of the Blake's pouch cyst (a) showing a small pore on the cyst wall. The choroid plexus of the fourth ventricle protrudes into the cyst cavity. vermis $(v)$, choroid plexus $(c)$, medulla oblongata $(m)$, fenestration on the cyst wall (arrowhead). Pathology

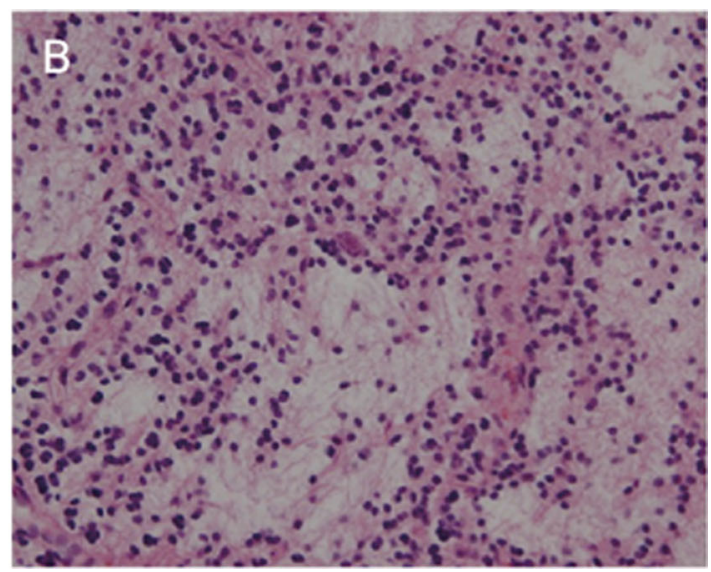

specimen (b) stained with hematoxylin and eosin revealing a markedly mucoid matrix, monomorphous bipolar cells, and an angiocentric cell arrangement. Tumors were positive for GFAP but negative for EMA (not shown). Ki-67 labeling indices were found to be $5 \%$ (not shown) 




Fig. 4 Two possible mechanisms for Blake's pouch cyst development. Anterior membranous area $(A M A)$, choroid plexus of the fourth ventricle $(C)$, cerebellum forming $(C e)$, midbrain $(M)$, posterior membranous area $(P M A)$, the fourth ventricle $(I V)$. In normal subjects, the choroid glomus is formed from the AMA $(\mathbf{a}, \mathbf{b})$. Then the PMA expands posteriorly to form Blake's pouch (c), which disappears leaving a small metapore, the future

resulted in re-expansion of the pouch and the onset of hydrocephalus. Considering our case, the intraoperative observation confirmed that the foramina of Luschka was open without any membranous structure to block the CSF outlet from the foramina to the subarachnoid space. Therefore, the widely disseminated tumor in the ventricle's wall might impair the transventricular absorption of CSF through the brain parenchyma, which resulted in stagnated CSF and finally induced re-expansion of the BPC. Some past cases, excluding the prenatally or neonatally diagnosed cases, which may be classified as classic congenital BPC, were exposed to a trigger altering the CSF environment, such as meningitis [4] or an association of syringomyelia [9]. This hypothesis explains why BPC, originally thought as congenital, is sometimes found in adults or even the elderly. However, this is not certain and should be validated in a much larger series of cases.

Although the standard surgical treatment of hydrocephalus associated with BPC has not yet been established, it is more commonly managed by ETV, which shows a relatively good outcome with minimal side effects even in neonates and infants $[4,10]$. We initially performed ETV, but the subsequent bacterial meningitis exacerbated the efficacy of ETV, and finally necessitated the shunt. Reviewing the pathogenesis of our patient's hydrocephalus retrospectively, compared with the meningitis, the multiple tumors inducing communicating hydrocephalus were a major cause, and the hydrocephalus was finally managed by the shunt. Therefore, it is important to exclude the underlying mechanism of BPC-associated hydrocephalus, whether it is a simple problem of CSF obstruction at the BPC that can be managed by ETV or a concomitant factor of communicating hydrocephalus, which may need a shunt. foramen of Magendie (d). The congenital or classic Blake's pouch cyst develops if the small metapore does not form, and Blake's pouch persists. In some cases, such as the current case, the original Blake's pouch almost disappears leaving a small remnant of its cyst wall. This remnant may reexpand to develop a postnatal or secondary Blake's pouch cyst as a result of some trigger. See text for more details. (Modified from [2])

In summary, this report of a rare but important case provides evidence that BPC might develop after birth following a change in CSF dynamics, suggesting that BPC may be classified to two subgroups: a classic congenital subgroup and a postnatal secondary subgroup. We speculate that asymptomatic BPC, cases with a remnant Blake's pouch or re-expanded BPC with no CSF alterations, may be more common than previously thought.

\section{References}

1. Blake JA (1900) The roof and lateral recesses of the fourth ventricle, considered morphologically and embryologically. J Comp Neurol 10: 79-108

2. Tortori-Donati P, Fondelli MP, Rossi A, Carini S (1996) Cystic malformations of the posterior cranial fossa originating from a defect of the posterior membranous area. Mega cisterna magna and persisting Blake's pouch: two separate entities. Childs Nerv Syst 12:303-308

3. Calabro F, Arcuri T, Jinkins JR (2000) Blake's pouch cyst: an entity within the Dandy-Walker continuum. Neuroradiology 42:290-295

4. Cornips EM, Overvliet GM, Weber JW, Postma AA, Hoeberigs CM, Baldewijns MM, Vles JS (2010) The clinical spectrum of Blake's pouch cyst: report of six illustrative cases. Childs Nerv Syst 26:10571064

5. Lafouge A, Gorincour G, Desbriere R, Quarello E (2012) Prenatal diagnosis of Blake's pouch cyst following first-trimester observation of enlarged intracranial translucency. Ultrasound Obstet Gynecol 40: 479-480

6. Garel C, Moutard ML (2014) Main congenital cerebral anomalies: how prenatal imaging aids counseling. Fetal Diagn Ther.

7. Gardiner K, Chitayat D, Choufani S, Shuman C, Blaser S, Terespolsky D, Farrell S, Reiss R, Wodak S, Pu S, Ray PN, Baskin 
B, Weksberg R (2012) Brain abnormalities in patients with Beckwith-Wiedemann syndrome. Am J Med Genet A 158A:13881394

8. Iuculano A, Zoppi MA, Ibba RM, Monni G (2014) A case of enlarged intracranial translucency in a fetus with Blake's pouch cyst. Case RepObstet Gynecol 2014:968089
9. Conti C, Lunardi P, Bozzao A, Liccardo G, Fraioli B (2003) Syringomyelia associated with hydrocephalus and Blake's pouch cyst: case report. Spine (Phila Pa 1976) 28:E279-E283

10. Brusius CV, Cavalheiro S (2013) Endoscopic third ventriculostomy is a safe and effective procedure for the treatment of Blake's pouch cyst. Arq Neuropsiquiatr 71:545-548 